Planetary Systems in the Universe - Observation, Formation and Evolution

Proceedings IAU Symposium No. 202, (c)2004 IAU

Alan Penny, Pawel Artymowicz, Anne-Marie Lagrange, 6 Sara Russell, eds.

\title{
HST Photometry of 47 Tucanae: Time Series Analysis and Search for Giant Planets
}

\author{
Timothy M. Brown \\ High Altitude Observatory/National Center for Atmospheric Research, \\ 3450 Mitchell Lane, Boulder, CO 80303, USA
}

David Charbonneau

HAO/NCAR and Harvard/Smithsonian Center for Astrophysics, 60 Garden St., Cambridge, MA 02138, USA

Ronald L. Gilliland

Space Telescope Science Institute, 3700 San Martin Dr., Baltimore, $M D$ 21218, USA

and M.D. Albrow (STScI), A. Burrows (U. Arizona), W.D. Cochran, N. Baliber (U. Texas), P.D. Edmonds (SAO), S. Frandsen, S. Bruntt (U. Aarhus), P. Guhathakurta, P. Choi, D.N.C. Lin, S.S. Vogt (UCSC), G.W. Marcy (UCB), M. Mayor, D. Naef (Obs. Geneva), E.F. Milone, C.R. Stagg, M.D. Williams (U. Calgary), A. Sarajedini (Wesleyan U.), S. Sigurdsson (PSU) and D.A. VandenBerg (U. Victoria)

Abstract. Gilliland et al. (2000) have reported HST photometric observations of 34000 stars in the globular cluster 47 Tuc, showing an absence of close-in giant planets in that cluster relative to their frequency in the solar neighborhood. Here we describe the methods of time-series analysis that were used to search the 47 Tuc data for transits by giant extrasolar planets, and the means by which these methods were validated.

\section{Introduction}

Roughly $1 \%$ of the Sun-like stars in the solar neighborhood are orbited by companions of approximately Jupiter's mass in orbits with semimajor axes smaller than 0.1 AU (Marcy, Cochran \& Mayor 2000). Because of the small orbital distances, these systems have a fair probability (about 10\%) of having orbits oriented so that transits occur, dimming the light of the star by one to a few percent for an interval of a few hours once each orbital period (typically 3-4 days). Indeed, one solar neighbor with a companion detected by radial velocity measurements (HD 209458) shows such transits (Charbonneau et al. 2000, Henry et al. 2000). Gilliland et al. (2000) used the exceptional photometric stability and high spatial resolution provided by the Hubble Space Telescope to search for such transits among 34000 stars in the globular cluster 47 Tuc. This search revealed no transiting planets, although projection from the occurrence rate in the solar neighborhood suggests that more than 15 such planets should 
have been detected. Central to the argument is an understanding of the sensitivity and reliability of the analysis methods used to search for transits in the HST time series. We give here a brief description of the methods used; a fuller description will be provided by Brown et al. (2001).

\section{Data Processing}

The time series of stellar brightnesses provided by the image analysis pipeline consisted of typically 6 brightness measurements in each of two colors (roughly $\mathrm{V}$ and I passbands) for each of the $120 \mathrm{HST}$ orbits (8.3 continuous days) of the observing period. The noise properties of these time series data are close to ideal, being well approximated by a white Gaussian noise model with RMS noise amplitudes that are only slightly larger than photon shot noise. Many of the stellar time series do, however, contain small noise contributions at the HST orbital frequency and its harmonics. As the first step in processing the timeseries data, we reduced the magnitude of these artifacts by means of a technique based on singular value decomposition of the arrays of residual relative brightness $v s$. star number and time.

Having removed most systematic noise signatures, we then searched each time series for evidence of transits and for other (more nearly sinusoidal) periodic signals. To detect transits, we applied a matched filter technique similar in conception to that described by Jenkins et al (1996), but much simpler in execution because it was restricted to the search for single planets orbiting single stars. Moreover, we assumed that the maximum transit duration (i.e., that for $\sin i=1$ ) for a given star could be accurately predicted given the star's mean magnitude (hence spectral type, hence radius) and an assumed orbital period. Thus, for each star it was necessary to search only a 2-dimensional space (orbital period and phase) for transit-like events. The search proceeded by folding the data with each trial orbital period, and then convolving the folded data string with the transit waveform being sought. The test waveform was normalized according to the total power in the individual time series, so that in the absence of a signal, the output of the convolution process was white Gaussian noise with unit variance. Possible transits were then indicated by positive peaks in the convolution with amplitudes greater than a chosen cutoff (we used $6.2 \sigma$ ). Because of the large number of statistical tests performed, a relatively high detection threshold was needed to avoid false detections. Even so, most detected events proved to result from isolated noise spikes. To discriminate against these, we analyzed data from the 2 colors separately, and required significant signals in both colors to confirm a detection.

To search for sinusoidal variations in stellar brightness, we simply computed the Scargle power spectrum of each time series, and searched for peaks in the spectra above a threshold of 12 times the mean power. In spite of the earlier SVD processing, many stars (particularly faint ones with bright neighbors) showed significant peaks at the HST orbital period or its harmonics. We rejected such power spectrum peaks as false detections.

Finally, we searched the list of possible detections for duplications between the transit-search and power spectrum lists, and for stars with identical periods and closely adjoining spatial positions. The latter search was necessary because 
cross-talk between nearby stars often mixed the signal from one true variable star into several of its fainter neighbors.

The process just described revealed many variable stars, including eclipsing binaries, pulsating variables, and rotating spotted stars. It did not, however, disclose any time series that could plausibly be identified as resulting from planetary transits.

\section{Validating the Data Analysis}

To assure that real signals of the expected size would have been seen, we added artificial transits to the time series and then processed these adulterated data in the normal way, to see if we could recover the input transit properties. We did this in two ways. The first of these was to modify a small number of stars in the calibrated 2-dimensional images; this assured that transits were not being lost in the photometry process. When these tests proved satisfactory, we added artificial transit signals to the time series of 10000 different stars, assuming a range of planetary radii, orbital periods, phases, and inclinations.

These tests showed that the fraction of detected transiting planets peaks for $\mathrm{V}$ magnitudes between 17 and 19 (bright stars have large radii, yielding small signals for a given size planet; faint ones have large photon noise). Detection sensitivity also depends strongly on planetary radius, falling below $50 \%$ at $\mathrm{V}=18$ for planetary radii below $1.1 R_{J u p}$. The detectability is slightly better for shorter periods than for longer ones (because more transits can be seen per unit observing time), but this effect is not strong. Given the distribution of $\mathrm{V}$ magnitudes in our sample, and assuming a typical orbital period of 3.5 days and planetary radii of $1.3 R_{\text {Jup }}$ (like HD $209458 \mathrm{~b}$ ), we reached the conclusion described above. See Gilliland et al. (2000) for a discussion of the astrophysical consequences.

This work was supported in part by STScI Grant GO-8267.01-97A to the Space Telescope Science Institute and several STScI grants from the same proposal to Co-I institutions.

\section{References}

Brown, T.M. et al. 2001, ApJ (in preparation)

Brown, T.M., Charbonneau, D., Gilliland, R.L., Noyes, R.W., \& Burrows, A. 2001, ApJ (submitted)

Charbonneau, D., Brown, T.M., Latham, D.W., \& Mayor, M. 2000, ApJ 529, L45

Gilliland, R.L. et al. 2000, ApJ (in press)

Henry, G.W., Marcy, G.W., Butler, R.P., \& Vogt, S.S. 2000, ApJ 529, L41

Jenkins, J.M., Doyle, L.R., \& Cullers, D.K. 1996, Icarus 119, 244

Marcy, G.W., Cochran, W.D. \& Mayor, M. 2000, in Protostars and Planets IV, ed. V. Mannings, A.P. Boss \& S. Russell, (Tucson: University of Arizona Press), 1285 